\title{
STRUCTURAL MODIFICATION OF CELLULOSE NANOCOMPOSITES BY STRETCHING
}

\author{
HITOSHI TAKAGI, ANTONIO N. NAKAGAITO \& YUYA SAKAGUCHI \\ Graduate School of Science and Technology, Tokushima University, Japan
}

\begin{abstract}
Recently cellulose nanofibers and their nanocomposites have attracted researchers' interest, because the mechanical performances of the cellulose nanofiber are high enough to use as reinforcement in polymer nanocomposites; for example, tensile strength is 2-3 GPa. However actual mechanical properties of polymer nanocomposites (i.e. green nanocomposites) reinforced by the cellulose nanofiber are much lower than expected. There are several reasons for the poor mechanical properties; such as fiber orientation and matrix/fiber bonding. In this study, we intended to improve the mechanical properties of polymer nanocomposites by controlling the orientation of cellulose nanofiber. The fiber orientation control of the cellulose nanofiber was conducted by applying multiple mechanical stretching treatments. In order to get higher stretching strain, we used polyvinyl alcohol gel as a matrix polymer. The effectiveness of the fiber alignment control has been demonstrated by tensile tests, namely the tensile strength and Young's modulus of the cellulose nanofiber-reinforced nanocomposites after stretching treatments were increased as compared with those of the untreated nanocomposites.

Keywords: cellulose, nanofiber, green composites, stretching, fiber orientation.
\end{abstract}

\section{INTRODUCTION}

Various kinds of bio-based composite materials have been developed until now [1]-[6]. Recently, cellulose nanofibers have drawn scientists' attention as one of potential bio-based nanoscale reinforcing fibers, because the cellulose nanofibers have excellent mechanical properties, e.g., tensile strength of about 2-3 GPa [7], and Young's modulus of about $140 \mathrm{GPa}$ [8]. These mechanical properties are equivalent to those of conventional glass and aramid fibers.

However, the mechanical properties of most cellulose nanofiber-reinforced polymeric composite materials are considerably lower than we expected. For example, the starch-based green composites reinforced by commercially available cellulose nanofiber have flexural strength of $68 \mathrm{MPa}$ and flexural modulus of $6.6 \mathrm{GPa}$ [9]. There are several reasons for this inferior mechanical property; one of possible reasons is a fiber alignment, i.e. random orientation of reinforcing cellulose nanofiber in the polymeric matrix [10].

In order to control the fiber alignment in polymer composites, magnetic field orientation control system as well as electric field orientation control system for carbon-based nanofillers was developed until now. In the electric field orientation control system, the fiber orientation of carbon nanotube was performed by applying strong electric field, and then fabricated epoxy-based polymer composite materials [11]. It was reported that the modulus in the normal direction of the orientation-controlled nanocomposites was lower than that of randomly oriented composites. Additionally, Kvien and Oksman reported that fiber orientation control for cellulose whisker was achieved by applying an electric field [12].

In our previous research work [10], the effectiveness of multiple extension treatment on the mechanical property of cellulose nanofiber-reinforced nanocomposites was demonstrated, however total strain introduced was limited, because of relatively poor deformation properties, namely lower fracture strain. In this study, therefore we used polyvinyl alcohol (PVA) gel as a matrix polymer, whose deformability is much higher than that of solid-PVA (namely non-gel PVA). In addition, we used grinder-treated cellulose nanofiber to check the 
size effect of reinforcing cellulose nanofiber. The effectiveness of the fiber alignment control in cellulose nanofiber-reinforced composite system was demonstrated experimentally; the mechanical performances of the cellulose nanofiber composites were enhanced after fiber alignment treatment by means of mechanical stretching treatments.

\section{EXPERIMENTAL METHODS}

\subsection{Materials and preparation of gel-PVA/CNF nanocomposite sheet}

PVA powder (162-16325, Wako Pure Chemical Industries Co., Ltd. Japan) was used as a biodegradable polymer matrix. Commercially available cellulose nanofiber (KY-100G, Co., Ltd. Daicel Co., Japan, hereafter CNF) was used as reinforcement. PVA powder was dissolved in hot water (about $80^{\circ} \mathrm{C}$ ), after $25 \mathrm{~g}$ of PVA was mixed with distilled water of 475g, and 5wt.\% PVA solution was prepared. After that, CNF of $1.5 \mathrm{~g}$ was mixed with distilled water of $135 \mathrm{~g}$ to prepare water suspension with $1 \mathrm{wt} . \%$ CNF. This water suspension and the PVA solution were mixed to make the PVA mixture having final CNF content of 10wt.\%. This PVA/CNF suspension was poured into a plastic container (i.e. casting treatment), and then quickly breezed it in a freezer. The frozen sheet was then defrosted by taking out of the freezer and leaving it at room temperature (about $20^{\circ} \mathrm{C}$ ). This freezing and defrosting treatments were repeated five times in total, and finally gel- PVA/CNF nanocomposite sheets were obtained.

\subsection{Grinding treatment}

Cellulose nanofiber suspension (solid content=1wt.\%) was prepared, and then fibrillated using a grinding machine (Supermasscolloider (Fig. 1), Masuko Sangyo Co., Ltd., Japan) at 1500 revolution per minute. This fibrillation treatment was repeated three times. Fig. 2 indicates the scanning electron micrographs of original CNF (KY-100G) and grinder-treated CNF (hereafter g-CNF).

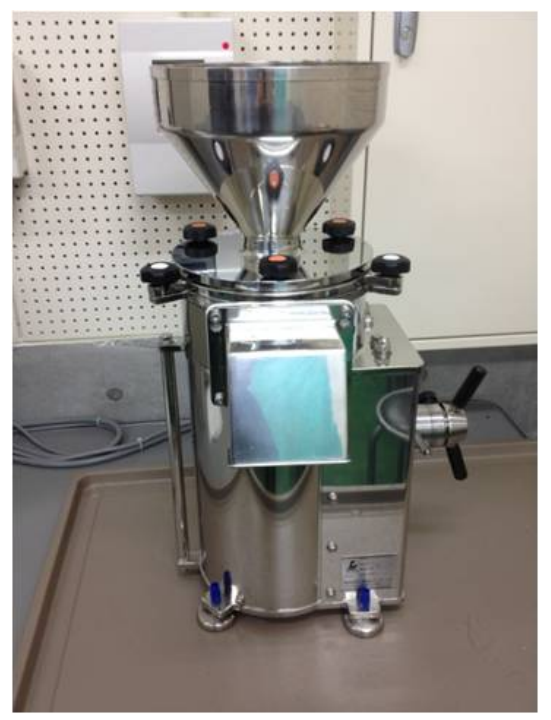

Figure 1: Supermasscolloider used in this study. 


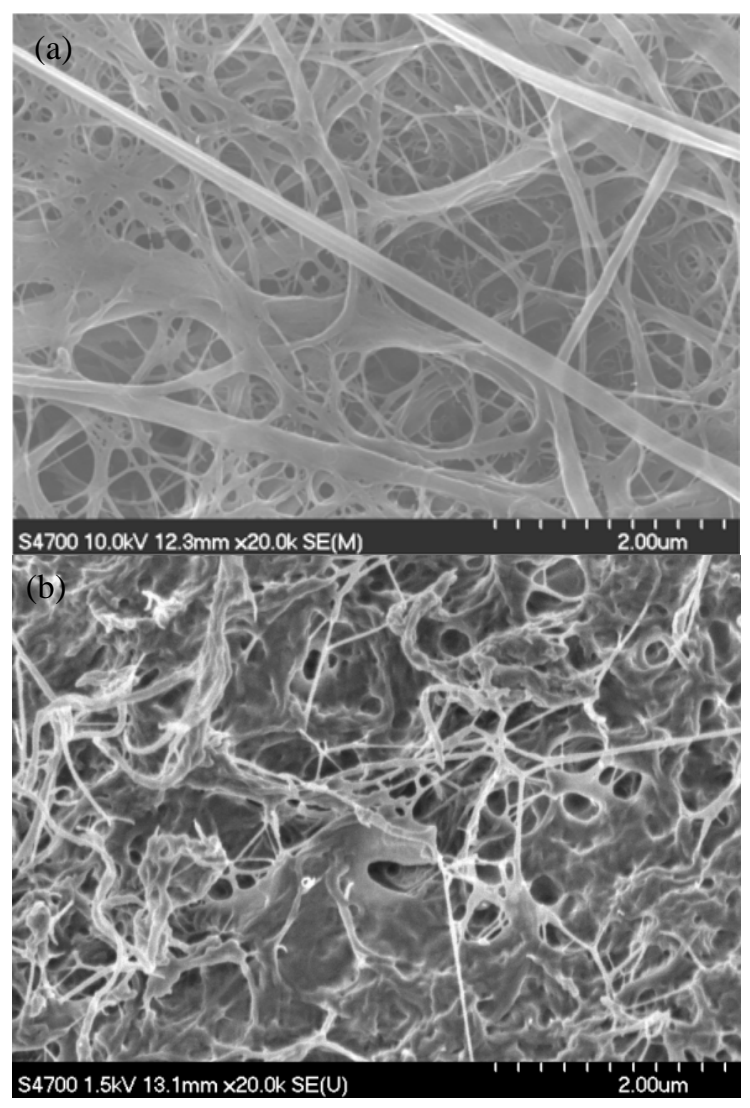

Figure 2: SEM photomicrographs of (a) CNF and (b) g-CNF used in this study.

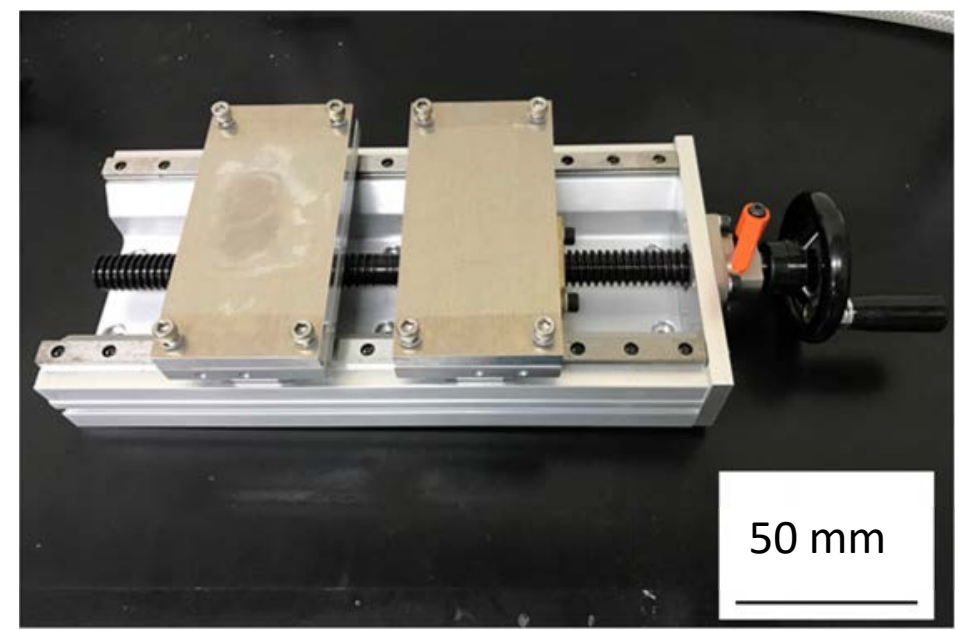

Figure 3: Photograph of mechanical stretching machine. 


\subsection{Mechanical extension treatment for gel-PVA/CNF nanocomposite sheets}

The gel PVA/10wt.\%CNF nanocomposite sheets were cut into rectangular stripes of $50 \mathrm{~mm} \times 120 \mathrm{~mm}$. This nanocomposite sheet was mechanically stretched by using a handmade stretching machine as shown in Fig. 3, and then dried for $24 \mathrm{~h}$. Finally, the nanocomposite sheet was hot-pressed at $15 \mathrm{MPa}$ and at $180^{\circ} \mathrm{C}$ for $10 \mathrm{~min}$. by a hot-pressing machine (IMC-16EF, Imoto Machinery Co. Ltd., Japan).

\subsection{Tensile testing of gel-PVA/CNF nanocomposite sheets}

The tensile test samples were rectangular-shaped with dimension of $50 \mathrm{~mm} \times 10 \mathrm{~mm}$. Tensile tests were carried out at a tensile speed of $1.0 \mathrm{~mm} / \mathrm{min}$ on an Instron universal testing machine (Model 5567, U.S.A.). The gauge length of the specimen was $30 \mathrm{~mm}$. Both tensile strength and Young's modulus were determined from the measured stress-strain curves.

\section{RESULTS AND DISCUSSION}

The results of tensile tests for gel-PVA/CNF nanocomposites and gel-PVA/g-CNF nanocomposites are indicated in Fig. 4 and Fig. 5, respectively. Tensile strength and Young's modulus of the both nanocomposites with and without stretching (stretching strain of $20 \%$ and $40 \%$ ) are also depicted in the same figure. As you can see from these graphs, gel-PVA/CNF nanocomposites are more ductile than solid PVA/CNF nanocomposites (maximum stretching strain was 5\%), whose mechanical properties have been reported elsewhere [10]. Considerable improvement on strength and Young's modulus is observed by applying the stretching treatment.

The mechanical properties of gel-PVA/CNF nanocomposites are improved after $40 \%$ stretching; i.e. tensile strength and Young's modulus are increased by $16 \%$ and $39 \%$, respectively, as compared with those of un-stretched nanocomposites. It can be seen that the mechanical properties also depend on testing direction. The tensile strength and Young's modulus of the nanocomposites tested along 90 degrees direction are lower than those of untreated nanocomposites, indicating that the effectiveness of mechanical stretching treatment.

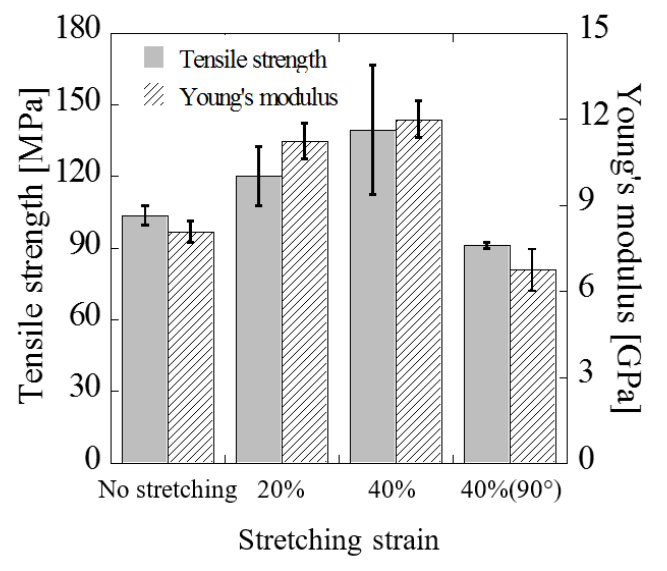

Figure 4: Variation of tensile strength and Young's modulus of gel-PVA/CNF nanocomposites; fiber content $=10 \mathrm{wt} . \%$. 


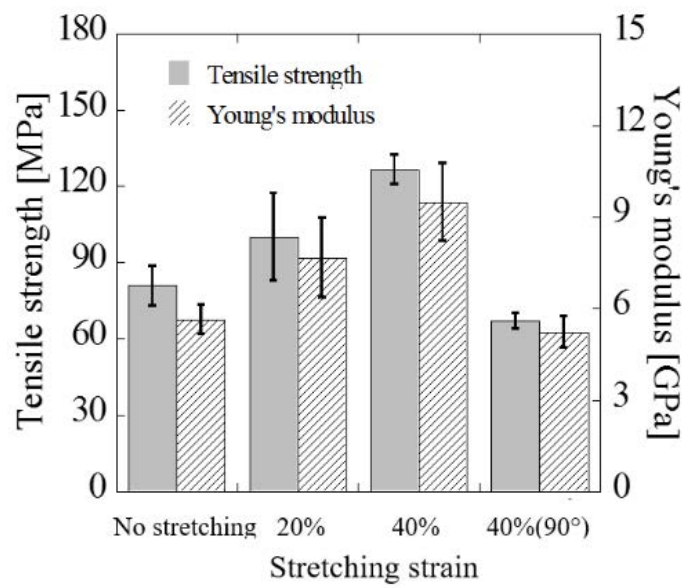

Figure 5: Variation of tensile strength and Young's modulus of gel-PVA/g-CNF nanocomposites; fiber content=10wt.\%.

The mechanical performance of gel-PVA/g-CNF nanocomposites was higher than that of gel-PVA/CNF nanocomposites. This results suggested that the ability of fiber orientation control in CNF nanocomposites became higher in the nanocomposites reinforced by thinner CNF; namely g-CNF.

\section{CONCLUSIONS}

The effects of mechanical stretching and fiber fibrillation treatments on the mechanical properties of gel-PVA/CNF nanocomposites and gel-PVA/g-CNF nanocomposites were investigated. From the results obtained in these investigations, the following conclusions were obtained.

- $\quad$ The gel-PVA/CNF nanocomposites can be applied much higher stretching strain than solid-PVA/CNF nanocomposites. The total stretching strain increased eightfold.

- The mechanical properties; such as tensile strength and Young's modulus, of the gel-PVA/CNF nanocomposites increased with increasing stretching strain.

- Both tensile strength and Young's modulus of gel-PVA/CNF nanocomposites, tested along 90 degrees direction, were lower than those of the un-stretched nanocomposites. This result supports the effectiveness of the mechanical stretching treatment in CNF-based nanocomposites.

- The increases in mechanical properties of stretched gel-PVA/g-CNF nanocomposites were higher than that of gel-PVA/CNF nanocomposites, suggesting that the ability of fiber orientation control in CNF nanocomposites became higher in thinner CNF-reinforced nanocomposites.

\section{ACKNOWLEDGEMENTS}

This work was supported by Japan Society for the Promotion of Science (JSPS) KAKENHI Grant Number $15 \mathrm{~K} 14148$ and the Ministry of Education, Culture, Sports, Science and Technology, Japan, the project S1311036. 


\section{REFERENCES}

[1] Cai, M., et al. Influence of alkali treatment on internal microstructure and tensile properties of abaca fibers. Industrial Crops and Products, 65, pp. 27-35, 2015.

[2] Liu, K., Takagi, H., Osugi, R. \& Yang, Z., Effect of physicochemical structure of natural fiber on transverse thermal conductivity of unidirectional abaca/bamboo fiber composites. Composites Part A: Applied Science and Manufacturing, 43(8), pp. 1234-1241, 2012.

[3] Pandey, J.K., Takagi, H., Nakagaito, A.N., Saini, D.R. \& Ahn, S.H., An overview on the cellulose based conducting composites. Composites Part B: Engineering, 43(7), pp. 2822-2826, 2012.

[4] Pandey, J.K., Lee, S., Kim, H.J., Takagi, H., Lee, C.S. \& Ahn, S.H., Preparation and properties of cellulose-based nano composites of clay and polypropylene. Journal of Applied Polymer Science, 125(S1), pp. E651-E660, 2012.

[5] Liu, K., Takagi, H. \& Yang, Z., Dependence of tensile properties of abaca fiber fragments and its unidirectional composites on the fragment height in the fiber stem, Composites Part A: Applied Science and Manufacturing, 45(2), pp. 14-22, 2013.

[6] Dong, Y., Bickford, T., Haroosh, T.J., Lau, K.T. \& Takagi, H., Multi-response analysis in the material characterisation of electrospun poly (lactic acid)/halloysite nanotube composite fibres based on Taguchi design of experiments: fibre diameter, non-intercalation and nucleation effects. Applied Physics. A, Materials Science \& Processing, 112(3), pp. 747-757, 2013.

[7] Sakurada, I., Nukushima, Y. \& Ito, T., Experimental determination of the elastic modulus of crystalline regions in oriented polymers. Journal of Polymer Science, 57(165), pp. 651-660, 1962.

[8] Page, D.H. \& EL-Hosseiny, F., The mechanical properties of single wood pulp fibres, Part VI Fibril angle and the shape of the of the stress-strain curve. Journal of Pulp and Paper Science, 9(4), pp. 99-100, 1983.

[9] Takagi, H. \& Asano, A., Effects of processing conditions on flexural properties of cellulose nanofiber reinforced "green" composites. Composites Part A: Applied Science and Manufacturing, 39(4), pp. 685-689, 2008.

[10] Takagi, H., Nakagaito, A.N., Nishimura, K. \& Matsui, T., Mechanical characterisation of nanocellulose composites after structural modification. WIT Transactions on The Built Environment, vol. 66, pp. 335-341, 2017.

[11] Hilding, J.M., Hong, M.P.L. \& Grulke, E.A., Alignment of dispersed multiwalled carbon nanotubes in low strength AC electrical fields. Journal of Nanoscience and Nanotechnology, 5(5), pp. 742-746, 2005.

[12] Kvien, I. \& Oksman, K., Orientation of cellulose nanowhiskers in polyvinyl alcohol. Applied Physics A-Materials Science \& Processing, 87(4), pp. 641-643, 2007. 